\title{
Quarter-sweep iterative alternating decomposition explicit algorithm applied to diffusion equations
}

\begin{abstract}
The aim of this article is to describe the formulation of the quarter-sweep iterative alternating decomposition explicit (QSIADE) method using the finite difference approach for solving one-dimensional diffusion equations. The concept of the QSIADE method is inspired via combination between the quarter-sweep iterative and the iterative alternating decomposition explicit (IADE) methods known as one of the technique in two-step iterative methods. The QSIADE method has been shown to be very fast as compared with the standard IADE method. Some numerical tests were included to support our statement.
\end{abstract}

Keyword: Diffusion equation; Full-, half- and quarter-sweeps iterative alternating decomposition explicit methods; Performance evaluation 